\title{
Wrist Rehabilitation Exoskeleton Robot based on Pneumatic Soft Actuators
}

\author{
Hassanin Al-Fahaam \\ Department of Robotics \\ University of Salford \\ Manchester, United Kingdom \\ h.s.h.al-fahaam@edu.salford.ac.uk
}

\author{
Steve Davis \\ Department of Robotics \\ University of Salford \\ Manchester, United Kingdom \\ s.t.davis@salford.ac.uk
}

\author{
Samia Nefti-Meziani \\ Department of Robotics \\ University of Salford \\ Manchester, United Kingdom \\ s.nefti-meziani@salford.ac.uk
}

\begin{abstract}
The aim of this paper is to describe the design of a soft, wearable splint for wrist joint rehabilitation, based on pneumatic soft actuators. The extensor bending and the contraction types of pneumatic soft actuators have been adopted in this study. These actuators are shown to be appropriate by examining their characteristics. The main contributions of this study are developing a safe, lightweight, soft and small actuator for direct human interaction, designing a novel single portable wearable soft robot capable of performing all wrist rehabilitation movements, and using low-cost materials to create the device. Three modes of rehabilitation exercises in the exoskeleton are involved: Flexion/Extension, Radial/Ulnar deviation, and circular movements.
\end{abstract}

Keywords- Wearable Robot; Human-friendly robot; Soft actuators; soft robotic; artificial pneumatic rubber muscle; Wrist Rehabilitation; Wrist Exoskeleton

\section{INTRODUCTION}

Stroke is one of the main sources of death worldwide and of disability in the developed countries [1]. In a study of the last decade, the American Heart Association mentioned that more than 795,000 persons in America suffer a stroke every year [2]. In a comparative study done in the same year, the Health Organization of Canada revealed that 1.3 million persons in Canada were diagnosed with a coronary illness and around 300,000 persons in Canada were living with poststroke impacts [3]. Following a stroke, most survivors suffer from numerous kinds of disabilities such as the inability to control their limbs in the activities of daily living. The upperlimbs is the most common disability among stroke patients [4]. Stroke survivors have neurological damage that results in loss of or weakness in capability to control their limbs. One of these losses is wrist functional motor capabilities. To treat these disabilities, patients must undergo long term rehabilitation.

Repetitive movement exercise is considered an effective kind of rehabilitation for patients with post-stroke impairments [5-10]. The aim of disabled patients' rehabilitation is to empower them for independent living and to assist them to be as productive as possible. This is done by developing a strong, flexible, soft, lightweight, balanced and safe rehabilitation device placed in the injured limb segment. Research has shown that the duration, capacity, and intensity of repetitive movement exercises have a huge impact on the motor rehabilitation improvement [11]. However, the long-term rehabilitation process may be a problem for numerous patients for many reasons. For example, individuals living far from the rehabilitation clinic may find it extremely difficult to travel frequently. Additionally, the long-term rehabilitation process may be too expensive for many patients. Therefore, patients may choose to stop the rehabilitation treatment, and thus lose their opportunity for an independent life. All these reasons have inspired the researchers to develop innovative rehabilitation devices and tools that can be portable and easy to use at home independently without therapist help.

In this paper, we present our wearable exoskeleton robot for wrist rehabilitation. Pneumatic soft actuators are used as wrist exoskeleton muscles to assist rehabilitation exercises. Both extensor bending and contraction types of pneumatic soft actuators have been developed. Most actuator characteristics have been achieved, such as pressure to length, bending angle, and force. Our system aims to fit any adult without needing to be changed mechanically. The exoskeleton is capable of performing all wrist rehabilitation movements.

\section{Pneumatic ARtificial Muscles}

The Pneumatic Muscle Actuator [12], also named the McKibben Pneumatic Artificial Muscle (PAM), Fluidic Actuator or Biomimetic Muscle, is a tube-like actuator that is characterized by a decrease or increase in the muscle length when pressurised [13]. The most common soft actuator is the McKibben Muscle, which was invented by the physician Joseph L. McKibben in the 1950s and was used as an orthotic appliance for polio patients [14].

The first commercial version of PAM was manufactured by a Japanese company named Bridgestone in the 1980s. These muscles are significantly lightweight actuators that feature smooth, fast, and accurate response and are also capable of producing high force when fully pressurised [13].

PAMs are typically designed and manufactured as a latex or rubber tube, surrounded by a braid sleeve [15-17]. Fiber wrapping surrounds the rubber tube for protection, and suitable plastic or metal fittings are attached to both ends. The PAMs convert pneumatic power to pulling/pushing force and also have many benefits, such as high force to weight ratio, variable installation possibilities, no requirement for mechanical parts, low consumption of compressed air and 
manufacture from cheap materials. Replacing heavy rigid motor parts, which cause much of the weight of a robot actuator, with lighter McKibben artificial muscles, will produce advantages in industrial and medical robots. The PAM is a pneumatic soft actuator, which shows numerous features found in real muscle.

\section{WRIST EXOSKELETON}

Exoskeleton power assistance and rehabilitation must be: i) safe because it is in direct interaction with humans, ii) lightweight, for easy use and portability, and iii), small and soft, to be flexible in daily usage. All these properties are found in pneumatic soft actuators, and therefore, many researchers depend on these soft actuators to manufacture power assistive and rehabilitation exoskeletons.

\section{A. Kinematics Of Wrist Motion}

The biomechanics of the wrist joint are more complex than the resulting movements of the wrist would suggest. The wrist movements of interest are illustrated in Fig. 1, and the following points explain the Flexion/Extension, Radial/Ulnar deviation bending angles [18].

- Extension: 0 to 70 degrees

- Flexion: 0 to 90 degrees

- Radial Deviation: 0 to 20 degrees

- Ulnar Deviation:0 to 50 degrees
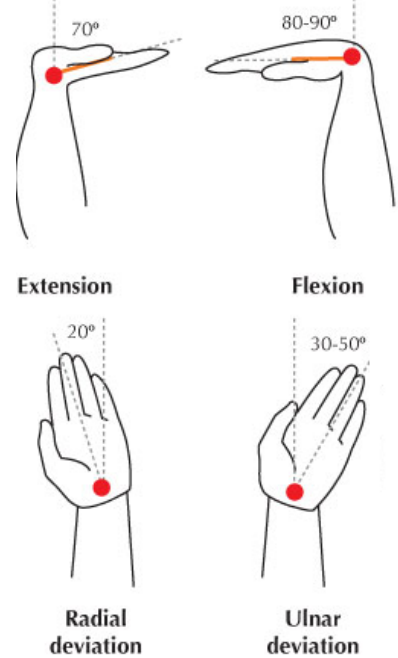

Fig. 1. Kinematics Of Wrist Motion.

\section{B. The Proposed Actuators}

The general design consists of an expandable bladder such as a rubber tube surrounded by a braided sleeve made of fibre threads which are attached to both sides. The muscles are available in many sizes, producing variable output forces, and the range of actuator displacement and also the lengths of muscles can be from lower than $10 \mathrm{~cm}$ up to $400 \mathrm{~cm}$ and the range of diameters from under $10 \mathrm{~mm}$ up to $70 \mathrm{~mm}$. Figure 3.2 shows the PMA construction [19].

\section{1) The contraction artificial muscles:}

Expansion of the rubber tube bladder against the braided sleeve occurs when pressure is applied. The braided sleeve acts to limit the expansion of the inner tube in order to maintain a cylindrical shape. When the pressure is increased, the volume of the inner tube increases in relation to the applied pressure, the contracting artificial muscle shortens and provides a pulling force to a mechanical load due to the extensor producing a pushing force. This basic principle effects the conversion of the radial stress on the rubber tube into axial stress and during relaxation of the muscle the reverse happens. A thin rubber tube transmits the applied pressure acting on it to the non-stretchable outside braid. Loads can be attached at one end of the PAM and the other end is for the air flow from the valve, as shown in Fig. 2.

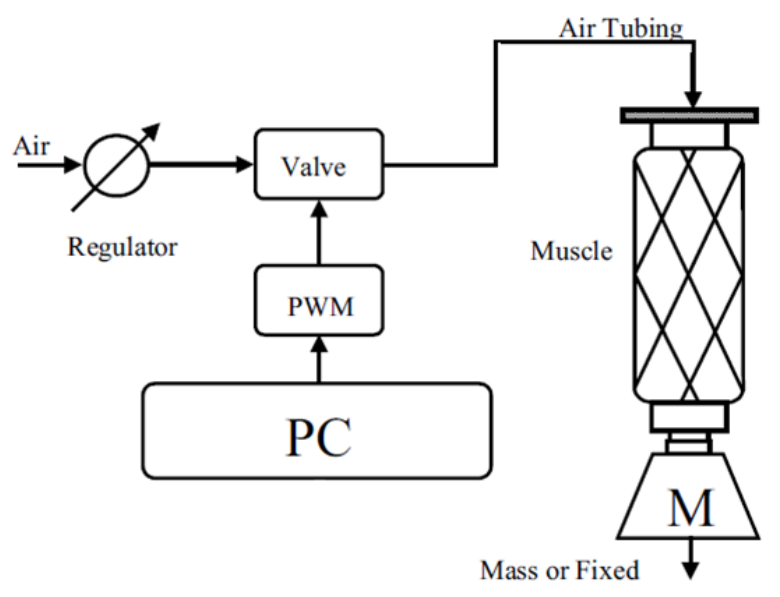

Fig. 2. The operation of PAM.

The proposed contraction muscle with different applied pressures is shown in Fig 3. The characteristics of this muscle are $20 \mathrm{~cm}$ sleeve braid length and $10 \mathrm{~mm}$ in radius; the bladder consists of double layers 350q Qualatex modelling latex balloon, $20 \mathrm{~cm}$ length and $10 \mathrm{~mm}$ radius with two terminals, one with a closed end.

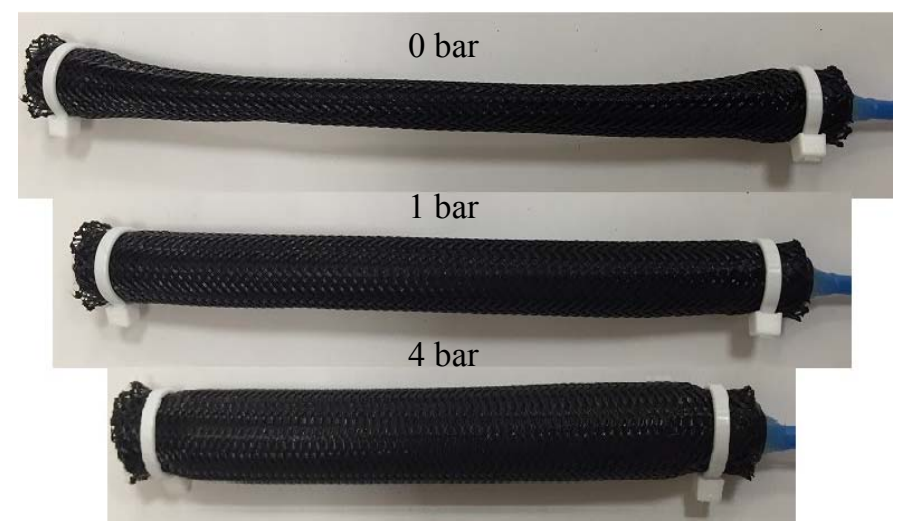

Fig. 3. The Proposed Contraction Muscle.

2) The extensor artificial muscles:

The artificial extensor muscle has the same construction as the contraction muscle, but the sleeve length is more than the 
bladder tube, in this case, it is $50 \mathrm{~cm}$. The proposed contraction muscle with different applied pressures is shown in Fig 4. It is clear that the extensor muscle length is increased in relation to the applied pressure.

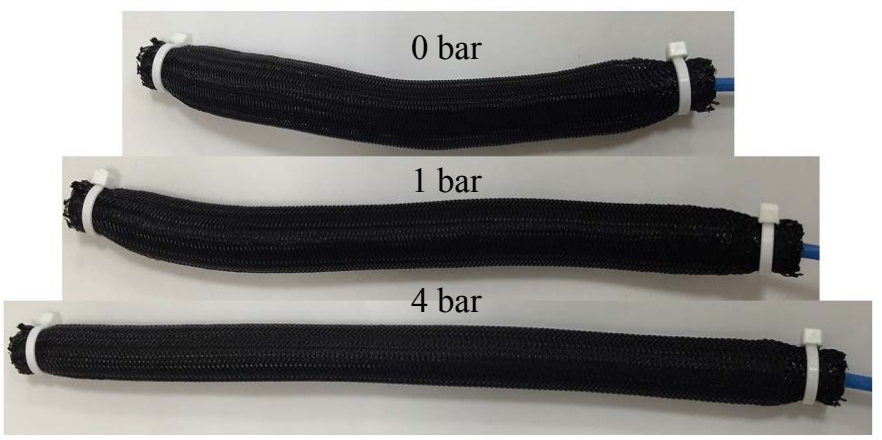

Fig. 4. The Proposed Extensor Muscle.

Fig. 5 shows the effect of increased and decreased applied pressure on the proposed contraction and extensor muscles. It can be seen from the curves that the shortest length of the contraction muscle at 5 bar is $13 \mathrm{~cm}$, then the percentage of contraction is $30 \%$, and the maximum length of the of the extensor muscle is at 5 bar $30 \mathrm{~cm}$, then the percentage of the extensor is $50 \%$. The small difference between the filling and venting curves is because of the rubber bladder type (expansion in the rubber tube needs time to return to the previous size before filling).

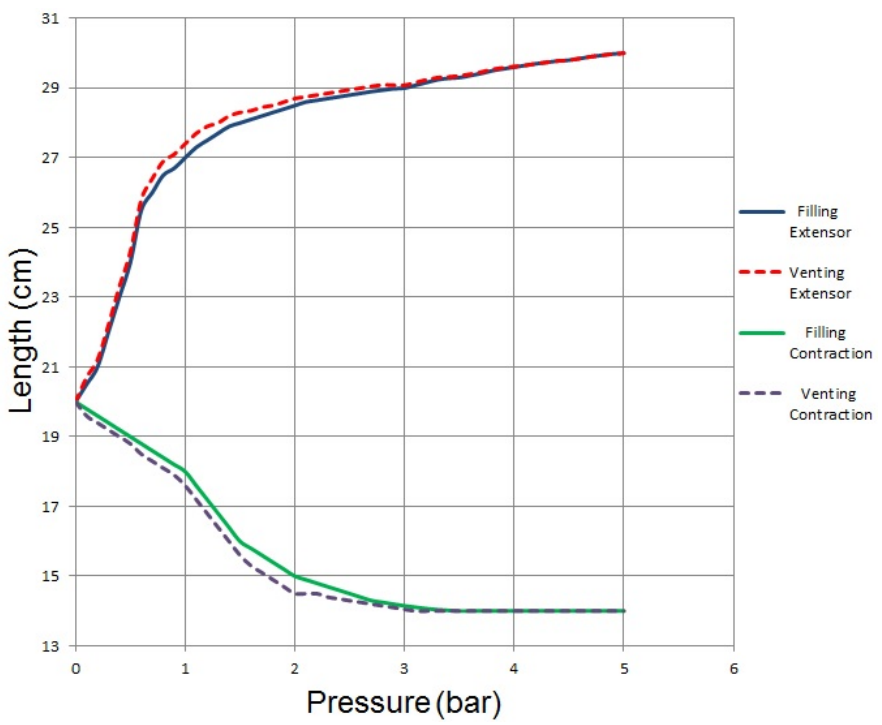

Fig. 5. The relationship between pressure and muscle length.

\section{3) The extended curved artificial muscle:}

We construct the extended curved muscle from the artificial extensor muscle by reinforcing one side of it with strong thread; as a result, the muscle will bend by increasing the applied pressure. When the pressure is increased in a curved extensor muscle, the bending angle increases in relation to the increase in the applied pressure. In other words, when the rubber tube is pressurized, only the top side is extended by the effect of reinforcement of the bottom side then the bending is occurred. Fig. 6 illustrates the curved type extensor muscle with different applied pressures; and Fig. 7 shows the characteristic relationship between the bending angle of a curved extensor muscle with increased and decreased applied pressure. The maximum bending angle of the curved muscle is approximately $220^{\circ}$ at 5 bar supplied pressure.

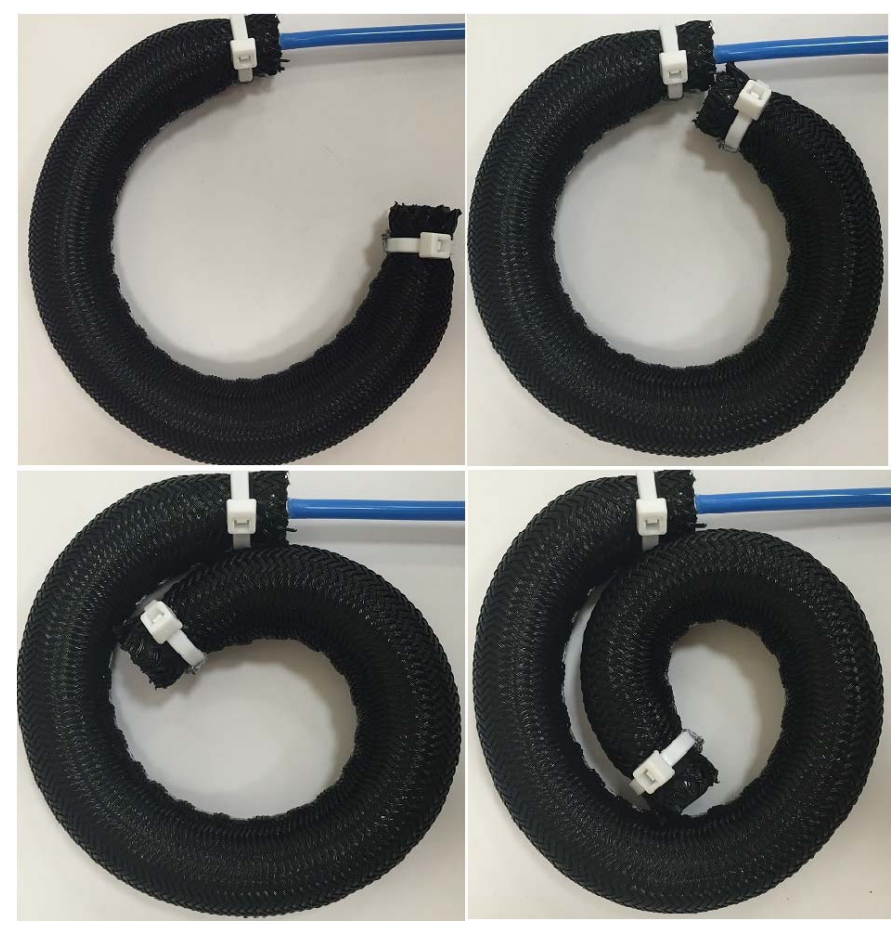

Fig. 6. Extended Curved Artificial muscle.

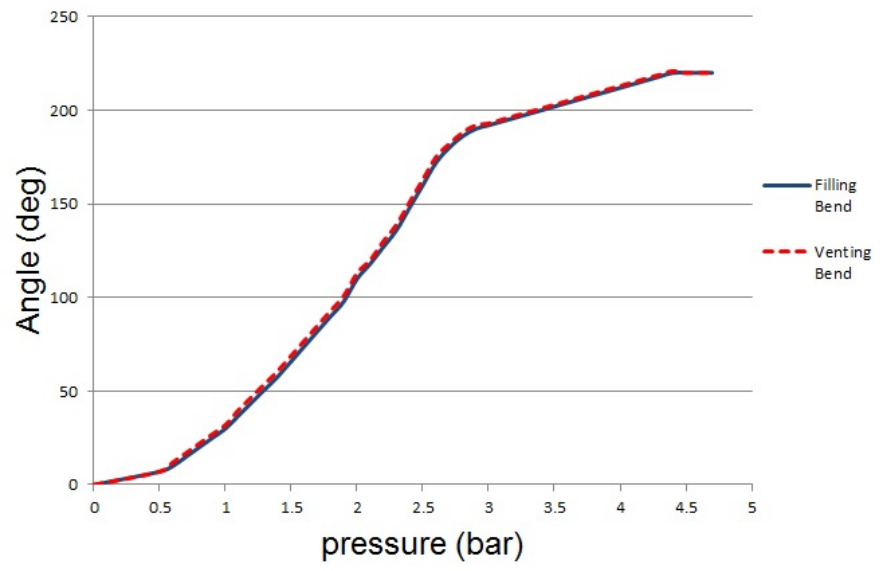

Fig. 7. Relationship between applied pressure and the angle of curved muscle.

\section{Exoskeleton Structure}

Fig. 8 shows a novel design of power assistive and rehabilitation exoskeleton for the wrist joint. Wrist flexion movement will be performed by the external assistive force from two extended bending muscles placed on the top side of the hand. Extension movement will be done by the external assistive pulling force from one contraction muscle placed on the same side of the Hand (between the two extended bending muscles). Radial and ulnar deviation movements will be performed by the external pulling forces from two contraction muscles placed on both sides of the hand. 
The structure of the wrist rehabilitation wearable robot is shown in Fig. 9 (a). It can be seen that the exoskeleton device consists of a traditional worker glove sewn to a medical power-plast compression wrap.

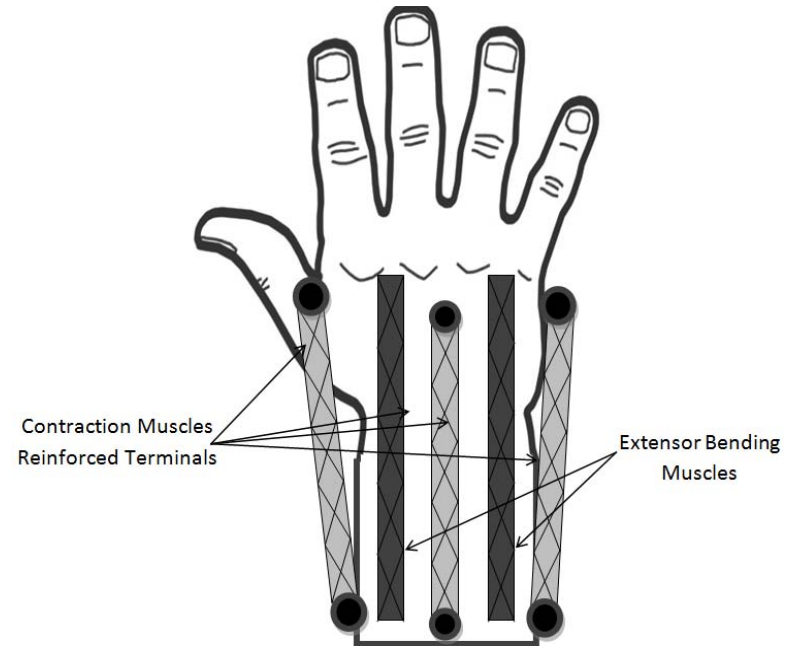

Fig. 8. The wrist exoskeleton design.

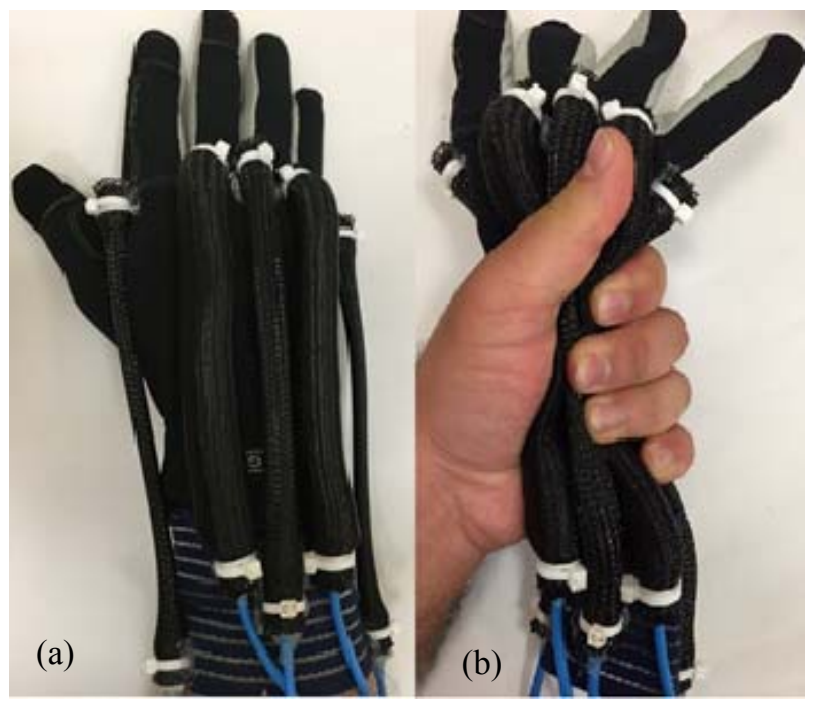

Fig. 9. The totally soft actuated wrist exoskeleton.

The medical power-plast compression wrap is used to reinforce the exoskeleton to prevent it slipping at the actuated stage. The same muscles in the previous section are used. The extended curved muscles are sewn along the top of the device starting from the finger roots. The contraction muscles are reinforced on their terminals at only one end, at the finger roots, and at the other ends on the medical power-plast. The extended curved muscles are supplied together from the same solenoid valve because they are always actuated together to perform the wrist flexion movement. The contraction muscles are each supplied from a separate solenoid valve because each one is actuated to perform different movements such as extension, radial deviation and ulnar deviation. Fig. 9 (b) shows that the exoskeleton is entirely soft and thus safe for direct human interaction. The total weight of the soft exoskeleton rehabilitation device is approximately $150 \mathrm{~g}$. Due to the light weight and the soft materials, it is portable, safe, flexible, fits any adult ( because it is made from stretched materials) and easy to use at home or work to perform the rehabilitation exercises.

\section{Exoskeleton Fundamental Characteristics}

\section{1) The Exoskeleton Output Force:}

The output force of each movement depends on the actuators and the supplied pressure. The wrist flexion movement force is the sum of the two extended bending muscles and the maximum force of this movement is approximately $37 \mathrm{~N}$. All other movements' output force is the same, because all of them use the characteristic contraction muscles and the maximum force is approximately $55 \mathrm{~N}$. Fig. 10 shows the exoskeleton output forces in relation to the applied pressure.

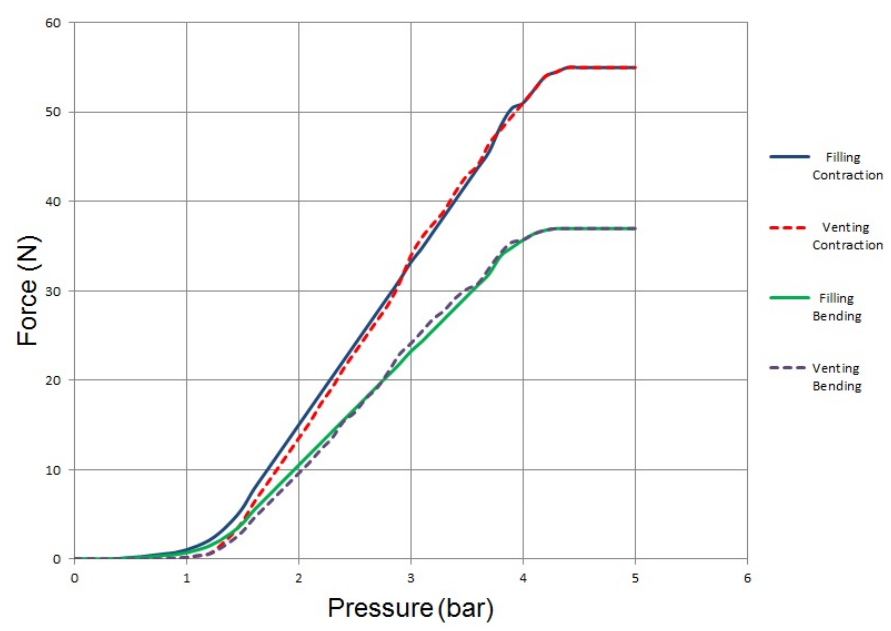

Fig. 10. The relationship between pressure and output force of exoskeleton.

\section{2) The Exoskeleton Rang of Motion:}

In wrist rehabilitation, there is a limited range of movements: flexion, extension, radial deviation and ulnar deviation, and circular movements. The main challenge is to perform all these movements by a single rehabilitation wearable robot without any help from a rehabilitation professional. A small, lightweight and easy to use device provides the capability of doing all rehabilitation exercises at home or anywhere instead of in a rehabilitation clinic. Fig. 11 shows the flexion and extension rehabilitation movements with assistance from the proposed prototype.

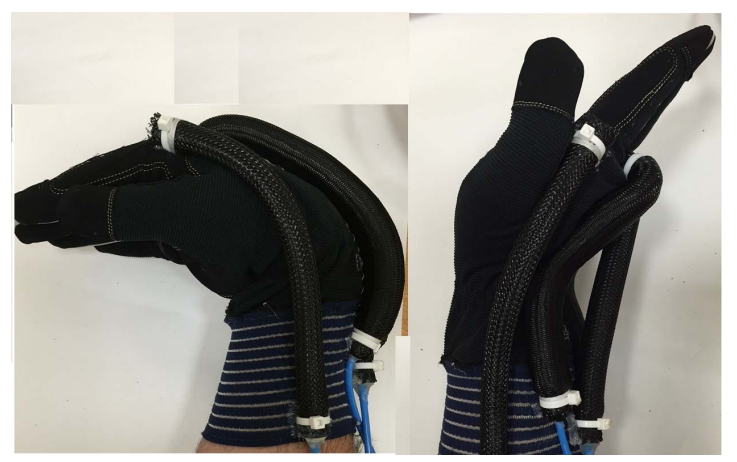

Fig. 11. Flexion and extension rehabilitation movements. 
Fig. 12 shows the radial deviation and ulnar deviation rehabilitation movements with assistance from the proposed prototype.

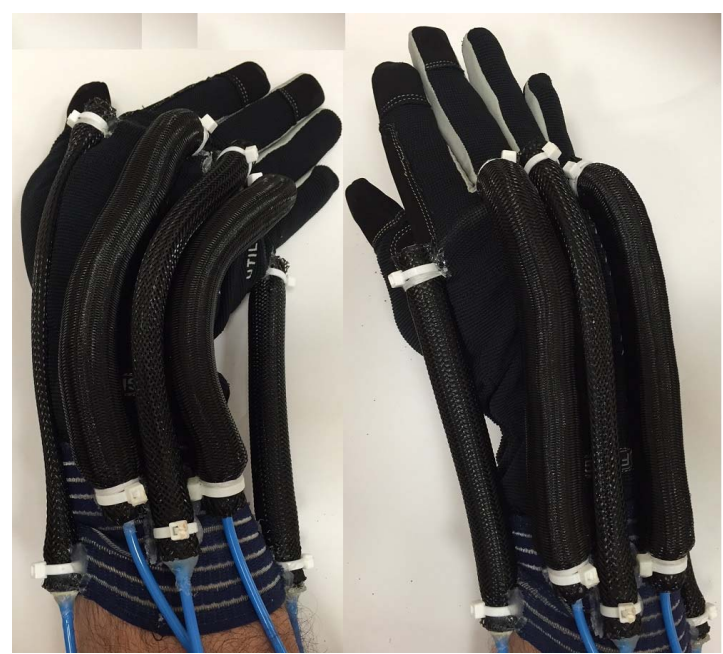

Fig. 12. Radial and ulnar deviation rehabilitation movements.

\section{3) The Exoskeleton Operation and Controller System:}

a) Controller System: Fig. 13 shows the block diagram of the exoskeleton controller system.

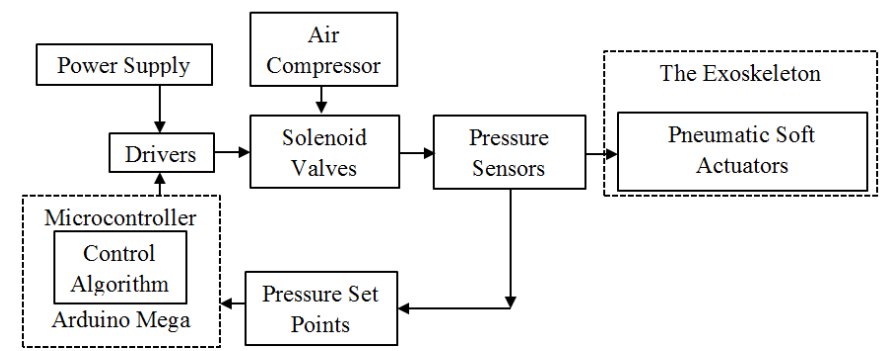

Fig. 13. Controller System.

b) Solenoid Valves: The air flow controlled by MATRIX 3/3 7504 channels series solenoid valves (see Fig. 14) [20]. The advantages of this valve are:

- compact dimension

- $\quad$ short response time

- insensitivity both to frequency work and to vibrations

- low absorbed power, precision, repetitiveness

- $\quad$ flexibility and long operation life.

c) The driver's circuit: the solenoid valve operates on 24 $\mathrm{v}$ pulse width modulation (PWM). The Arduino PWM output is only $5 \mathrm{v}$; the driver circuit is shown in Fig. 15. This circuit is repeated 8 times because the solenoid valve has 4 channels (4 filling +4 venting).

d) The pressure sensors: It is a Pressure sensor MDPS002 (700KPa) vacuum absolute pressure sensor (see Fig. 16). The output of this type of pressure sensor is microvolt, but the Arduino analog read pins sensitive range is $0-5 \mathrm{v}$. The INA122 precision instrumentation amplifier for accurate, low noise differential signal acquisition is used to amplify the pressure sensor signal.

e) The control algorithm: A direct control algorithm is used to control the exoskeleton. The pressure set points are from a variable potentiometer. This set point is easy to control manually to be appropriate from one patient to another to control the output force and bending angle for each exoskeleton movement.
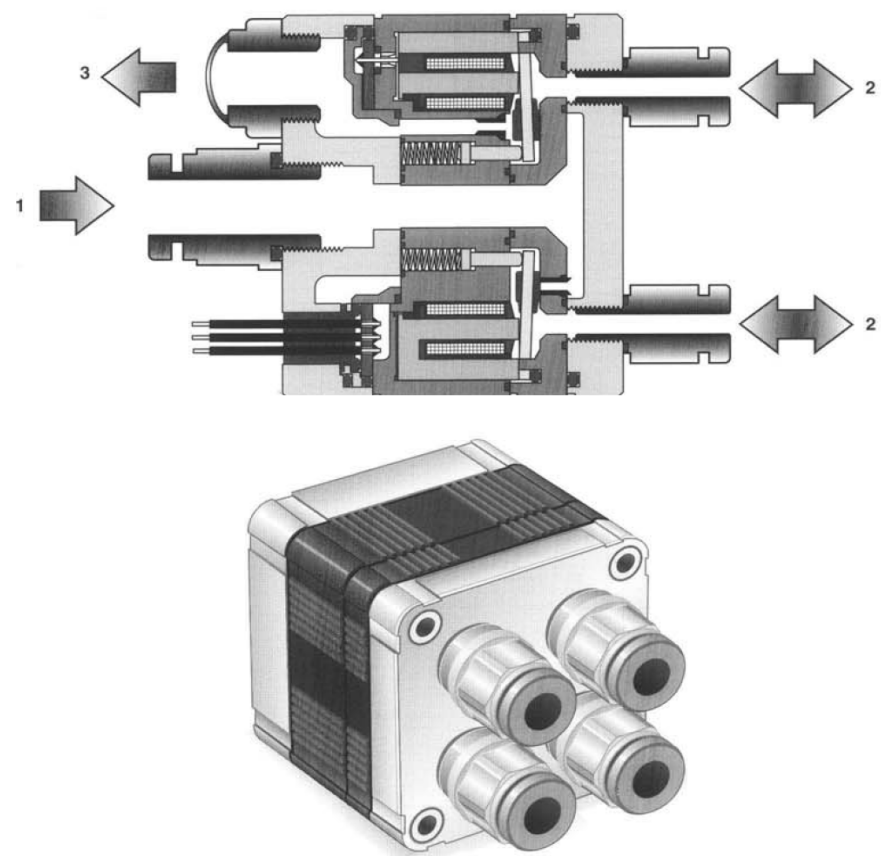

Fig. 14. MATRIX 3/3 750 series solenoid valve.

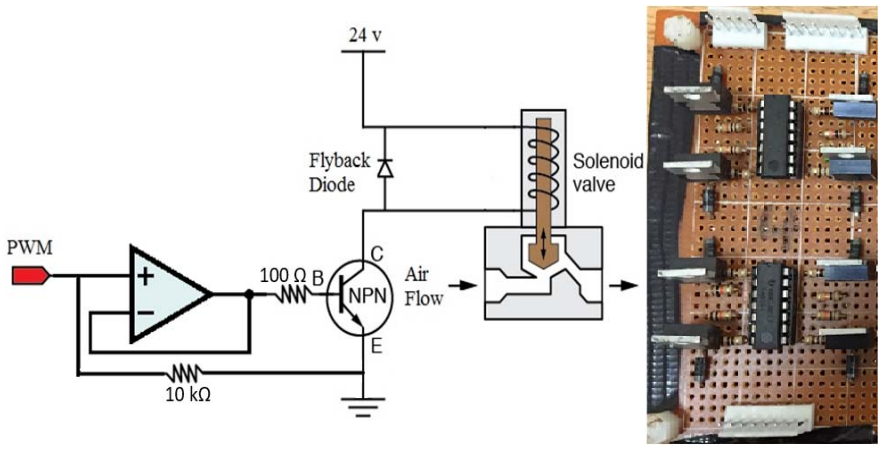

Fig. 15. The driver circuit.
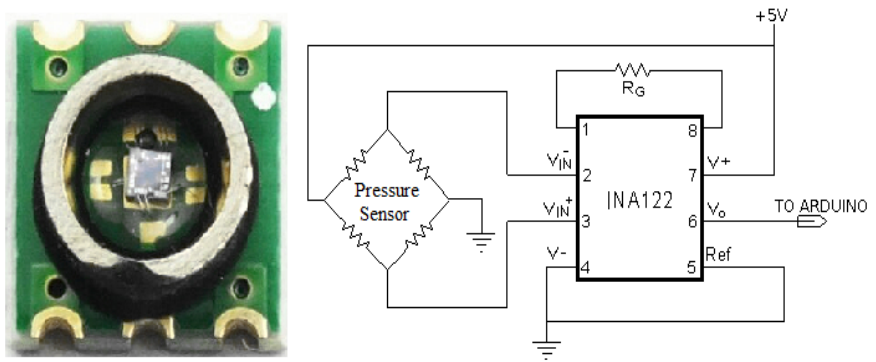

Fig. 16. The pressure sensor circuit. 


\section{CONCLUSION}

This study presents the preliminary stages of developing a wearable power assistive and rehabilitation glove based on pneumatic soft actuators. Curved extender and contraction artificial muscles are used to construct the soft exoskeleton. The exoskeleton aims to fit any adult without the design needing to be changed mechanically. The exoskeleton is capable of performing all wrist rehabilitation movements. The wrist flexion motion force is the sum of the two extended bending muscles and the maximum force of this movement is approximately $37 \mathrm{~N}$. All other movements' output force is the same because all of them use the characteristic contraction muscles and the maximum force is approximately $55 \mathrm{~N}$. The entire range of rehabilitation movements has been tested in this research. Future work is planned to develop the assistance to the elbow and improve the control algorithm. The range of rehabilitation movements will be increased. Android mobile applications will be developed to control the rehabilitation exercises.

\section{ACKNOWLEDGMENT}

The authors would like to thank the ministry of higher education/Iraq, University of Basrah, computer-engineering department for providing scholarship support to the first author of this paper.

\section{REFERENCES}

[1] C. J. Murray and A. D. Lopez, "Alternative projections of mortality and disability by cause 1990-2020: Global Burden of Disease Study," The Lancet, vol. 349, pp. 1498-1504, 1997.

[2] D. Lloyd-Jones, R. Adams, M. Carnethon, G. De Simone, T. B. Ferguson, K. Flegal, et al., "Heart disease and stroke statistics-2009 update a report from the American Heart Association Statistics Committee and Stroke Statistics Subcommittee," Circulation, vol. 119, pp. e21-e181, 2009.

[3] S. Dai, C. Bancej, A. Bienek, P. Walsh, P. Stewart, and A. Wielgosz, "Report summary Tracking heart disease and stroke in Canada 2009," Chronic Diseases and Injuries in Canada, vol. 29, 2009.

[4] J. W. Krakauer, "Arm function after stroke: from physiology to recovery," in Seminars in neurology, 2005, pp. 384-395.

[5] F. Just, K. Baur, R. Riener, V. Klamroth-Marganska, and G. Rauter, "Online adaptive compensation of the ARMin Rehabilitation Robot," in 2016 6th IEEE International Conference on Biomedical Robotics and Biomechatronics (BioRob), 2016, pp. 747-752.

[6] P. K. Jamwal, S. Hussain, M. H. Ghayesh, and S. V. Rogozina, "Impedance Control of an Intrinsically Compliant Parallel Ankle Rehabilitation Robot," IEEE Transactions on Industrial Electronics, vol. 63, pp. 3638-3647, 2016.

[7] D. Yun, A. M. Khan, R.-J. Yan, Y. Ji, H. Jang, J. Iqbal, et al., "Handling subject arm uncertainties for upper limb rehabilitation robot using robust sliding mode control," International Journal of Precision Engineering and Manufacturing, vol. 17, pp. 355-362, 2016.

[8] J. Wu, J. Gao, R. Song, R. Li, Y. Li, and L. Jiang, "The design and control of a 3DOF lower limb rehabilitation robot," Mechatronics, vol. 33, pp. 13-22, 2016.

[9] T. Tsuji, S. Itoh, S. Sakaino, and Y. Hasegawa, "Development of an upper limb rehabilitation robot with guidance control by pneumatic artificial muscles," in Mechatronics (ICM), 2015 IEEE International Conference on, 2015, pp. 426-431.

[10] B. Ugurlu, M. Nishimura, K. Hyodo, M. Kawanishi, and T. Narikiyo, "Proof of concept for robot-aided upper limb rehabilitation using disturbance observers," IEEE Transactions on Human-Machine Systems, vol. 45 , pp. 110-118, 2015.

[11] R. J. Nudo, B. M. Wise, F. SiFuentes, and G. W. Milliken, "Neural substrates for the effects of rehabilitative training on motor recovery after ischemic infarct," Science, vol. 272, p. 1791, 1996.

[12] D. Caldwell, G. Medrano-Cerda, and M. Goodwin, "Braided pneumatic actuator control of a multi-jointed manipulator," in Systems, Man and Cybernetics, 1993.'Systems Engineering in the Service of Humans', Conference Proceedings., International Conference on, 1993, pp. 423428.

[13] G. Andrikopoulos, G. Nikolakopoulos, and S. Manesis, "A survey on applications of pneumatic artificial muscles," in Control \& Automation (MED), 2011 19th Mediterranean Conference on, 2011, pp. 1439-1446.

[14] F. Daerden and D. Lefeber, "Pneumatic artificial muscles: actuators for robotics and automation," European journal of mechanical and environmental engineering, vol. 47, pp. 11-21, 2002.

[15] H. Aschemann and D. Schindele, "Comparison of model-based approaches to the compensation of hysteresis in the force characteristic of pneumatic muscles," IEEE Transactions on Industrial Electronics, vol. 61, pp. 3620-3629, 2014.

[16] K. Mortier, "Braided pneumatic muscles for rehabilitation apparatus," 2014.

[17] I. Veneva, B. Vanderborght, D. Lefeber, and P. Cherelle, "Propulsion System with Pneumatic Artificial Muscles for Powering Ankle-Foot Orthosis," Journal of Theoretical and Applied Mechanics, vol. 43, pp. 316, 2013.

[18] J. R. Celestino, "Characterization and control of a robot for wrist rehabilitation," Massachusetts Institute of Technology, 2003.

[19] S. Davis, N. Tsagarakis, J. Canderle, and D. G. Caldwell, "Enhanced modelling and performance in braided pneumatic muscle actuators," The International Journal of Robotics Research, vol. 22, pp. 213-227, 2003.

[20] R. W. Colbrunn, "Design and control of a robotic leg with braided pneumatic actuators," Case Western Reserve University, 2000. 